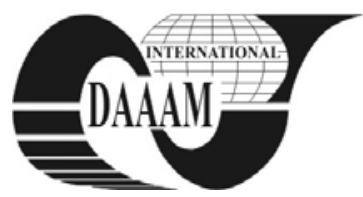

\title{
FINANCIAL TIME SERIES FORECASTING USING NEURO FUZZY APPROACH FOR THE BUCHAREST STOCK EXCHANGE
}

\author{
TRIFAN, A[lina] L[ucia]
}

\begin{abstract}
The motivation of this study is the research of an originally engineering field, but with important implications and applications for economics, in general and finance, in particular. Forecasting financial time series is the goal of many studies that combine concepts from various disciplines, in terms of classical theories or of the latest approaches, forming a point of great interest. Financial time series forecasting using neural networks, fuzzy systems, neuro fuzzy, genetic algorithms leads the research into the area of intelligent technology in an attempt to characterize the dynamic, hyperactive, catalyzing system of the capital markets, the behavior of the investors acting in this environment, the specific relationships.
\end{abstract}

Key words: fuzzy logic, neuro fuzzy systems, financial time series, forecasting, artificial neural networks

\section{INTRODUCTION}

The current business environment has become dependent on intelligent problem-solving techniques, continually being researched and developed methods and models in which the neural network rules are combined with genetic algorithms, fuzzy logic, neuro fuzzy systems and fuzzy expert systems.

Neural networks cannot be assinged an initial set of knowledge and have to follow a learning algorithm, a process depending on a time variable component that can not always ensure good results. Thus, the disadvantage of neural networks in terms of lack of transparency in the process of collecting, handling and processing of input data in output data led to the development of fuzzy expert systems, a special case of expert systems.

While neural networks have the information represented in the form of specific links, called weights, fuzzy systems are based on fuzzy logic, representing the information into fuzzy sets.

Fuzzy logic systems have the ability to interpret imprecise data and this is the reason why they can be useful in the decision making process. Handling and explaining inaccurate data and arguing decisions in linguistic form in the context of available factors is an advantage of fuzzy expert systems, but they can not automatically acquire the linguistic rules underlying the formulation of such decisions.

Neuro fuzzy systems are structures that combine in a hybrid intelligent technology the advantages of neural networks - their ability to learn and to adapt, to those of fuzzy logic - the ability of management of the human specific reasoning at linguistic level, transparency, interpretation of the generated models and handling uncertain data.

\section{LITERATURE REVIEW}

Fuzzy expert systems have been applied in numerous studies to solve various prediction problems (Bezdek, 1993; Bolloju, 1996; Kaneko, 1996; Al-Shammari \& Shaout, 1998; Kee, 2002; Yang et al., 2004; Huarng and Yu, 2005; Hassan et al., 2007; Tai-Liang Chen et al., 2008; Hakan , 2010).
Combining the learning abilities of the artificial neural networks and fuzzy logic, neuro fuzzy approaches have emerged. Many studies exploit hybrid neuro fuzzy systems, obtaining encouraging results and proposing different architectures (Gupta, 1994; Brown \& Harris, 1994; Pedrycz, 1995; Buckley \& Hayashi, 1995; Dash et al., 1995; Lie \& Sharaf, 1995; Studer \& Masulli, 1997; Padmakumari et al., 1999; Mitra \& Hayashi, 2000; Kulkarni, 2001; Lee et al., 2002; Craiger et al., 2003; Kim et al., 2004; Dušan, 2004; Radeerom et al., 2008; Atsalakis \& Valavanis, 2009; Venugopal et al., 2009; Chaudhuri et al., 2009; Jagric et al., 2010; Ebrahimpour et al., 2011).

\section{CASE STUDY}

\subsection{The structure of fuzzy logic systems}

A fuzzy logic system contains blocks, fuzzy sets used to sort incoming data by category, a process called fuzzification, decision rules that are applied to each set and a mechanism, called defuzzification to generate an output from the results of the rules.

In the fuzzification stage to each point (data) of each set is assigned a degree of membership (DOM) determined by the membership function, that may have different geometric shapes (bell-shaped, triangular, trapezoidal, singleton) and may be heterogeneous (e.g. fuzzy Markov chain).

Each rule inherits a degree of membership that is the result of the composition of the inputs degrees of membership. These rules may be introduced by a human factor before running the system or in the case of FAM model the rules are learned online.

Defuzzification is a procedure that creates a real non-fuzzy result by combining the results of the fuzzy rules.

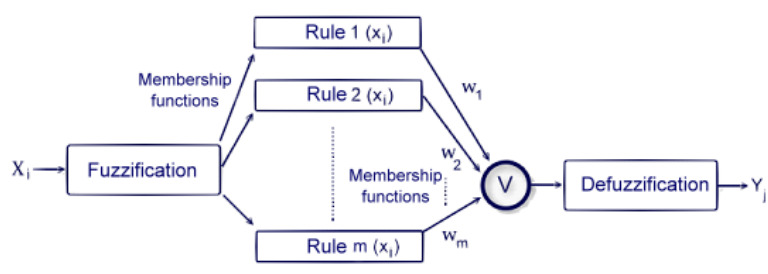

Fig. 1. General diagram of a fuzzy system

The two main steps of the inference process are aggregation and composition. Aggregation is a process of assessment of the rules from the previous part IF of the conditional instruction and composition is similar to the instruction conducted in a final decision instruction THEN.

During aggregation to each condition in the IF rule is assigned a degree of truth based on the degree of affiliation of the corresponding linguistic term. By applying the minimum function (min) or arithmetic operator product (prod) to the truth degrees of the conditions can be determined the degree of truth of IF part, that is assigned as degree of truth to the THEN part. 
Although systems based on fuzzy logic are getting good results on the forecast, the construction process of a fuzzy system is subjective and choosing the membership functions and rules for each scenario follows a heuristic pattern. Fixing the rules and selecting the membership functions in this manner are subject to intuition, trial and error.

\subsection{The hybrid neuro fuzzy model}

Having considered the advantages and disadvantages of artificial neural networks and fuzzy expert systems, the next step has resulted in the emergence of a hybrid model, combining the ability of learning, adaptation and generalization of neural networks with transparency and functionality of fuzzy systems, creating the hybrid neuro fuzzy systems.

Hybrid neuro fuzzy systems are endowed with specific modeling techniques of neural networks (the unique learning ability of a black-box type pattern) and of fuzzy logic structures (transparent interpretation and analysis of fuzzy IF-THEN rules).

With the ability to acquire and interpret linguistic IF-THEN rules by human experts, fuzzy neural networks with basis functions provide the architecture that combines in a uniform manner both numerical information and language skills.

Kosko (1992) proposed a remarkable pattern of neuro fuzzy system called FAM (Fuzzy Associative Memory), which involves the use of triangular membership functions and the scheme was implemented in the Trajectory Error Learning model as a feedforward component.

A FAM component contains two subcomponents: the rules of fuzzy logic and pattern relationships, the template entry/exit, called associative memory.

Three types of FAM have been used in various applications: Mamdani fuzzy model, Sugeno fuzzy model and fuzzy model of Tsukamoto.

\subsection{Building fuzzy logic and neuro fuzzy system}

Building a fuzzy logic system

The fundamental idea of building a fuzzy system is substituting numerical values specific to neural networks with linguistic terms features of the fuzzy logic that will be converted to quantitative signals belonging to the interval $[0,1]$.

The frame for building the indicators $\mathrm{K}, \mathrm{D}, \mathrm{KD}, \mathrm{KDM} 1$, KDP1 follows the methodology implemented in Trifan (2010).

\begin{tabular}{|l|c|c|l|}
\hline \multicolumn{3}{|c|}{ Linguistic variable } & \multirow{2}{*}{ Linguistic value } \\
\cline { 1 - 3 } Kame & Value & Type & \\
\hline D & & input & $\begin{array}{l}\text { very_small, small, } \\
\text { medium_small, } \\
\text { medium, } \\
\text { medium_high, } \\
\text { high, very_high }\end{array}$ \\
\cline { 1 - 2 } KD & {$[0,100]$} & input & negative, positive \\
\hline KDP1 & {$[-1,1]$} & output & $\begin{array}{l}\text { strong_decrease, } \\
\text { small_decrease, } \\
\text { stable, } \\
\text { small_growth, } \\
\text { strong_growth }\end{array}$ \\
\hline
\end{tabular}

Tab. 1. Properties of linguistic values and variables

\section{Neuro fuzzy approach}

The basic idea of a neuro fuzzy system is to determine the parameters of a fuzzy system using methods of learning specific to artificial neural networks.

In order to achieve this approach this study uses the FAM method, characterized by fuzzy rules with associated weights, allowing the error backpropagation (EBP) algorithm specific to backpropagation neural networks to combine with fuzzy logic. This technique is useful for generating and optimizing the membership functions and the weights associated with each rule in the data set.

\section{RESULTS}

\begin{tabular}{|c|c|c|c|c|c|}
\hline Model & $\mathrm{R}^{2}$ & $\bar{R}^{2}$ & $\mathrm{~h}(\mathrm{~N}=15144)$ & $\mathrm{HR}$ & $\mathrm{RMSE}$ \\
\hline $\begin{array}{c}\text { Linear } \\
\text { Regression }\end{array}$ & 0.7444 & 0.7432 & 12,160 & 0.8030 & 1.1010 \\
\hline ANN & - & - & 13,262 & 0.8757 & 0.8598 \\
\hline $\begin{array}{c}\text { Neuro } \\
\text { fuzzy }\end{array}$ & - & - & 13,781 & 0.9100 & 0.7807 \\
\hline
\end{tabular}

Tab. 2. Comparative average results for statistics and performance indicators

Even if models based on AI have achieved better results, consisting in superior values of performance compared to conventional models, they remain difficult to understand, apply and interpret, leaving open other possible options for better choices and combinations of parameters (learning rates, momentum, the choice of certain algorithms, linguistic variables, membership functions).

\section{CONCLUSION}

In the global crisis context the current financial climate hardly finds predictability in classical models, even in the latest approaches.

What stands out in favor of artificial neural networks, fuzzy logic, hybrid neuro fuzzy systems is their power to picture and replicate human features (learning, modeling, generalization, patterns, sorting).

The additional features of the individual categories that use artificial intelligence technology provide them the capacity to solve specific problems. Such capabilities and restrictions have caused advantages and disadvantages of using one or another existing technology and have created smart hybrid systems able to solve many complex problems.

\section{REFERENCES}

Atsalakis, G.S.; Valavanis, P. (2009). Forecasting stock market short-term trends using a neuro-fuzzy based methodology, Expert Systems with Applications, 36, pp. 10696-10707

Ebrahimpour, R.; Nikooc, H.; Masoudniad, S.; Yousefie, M.; Ghaemif, M.S. (2011). Mixture of MLP-experts for trend forecasting of time series: A case study of the Tehran stock exchange, International Journal of Forecasting, 27 (3), pp. 804-816

Hassan, R.; Nath, B.; Kirley, M.A. (2007). Fusion model of HMM, ANN and GA for stock market forecasting, Expert Systems with Applications, 33, pp. 171-180

Huarng, K.;Yu, H.K. (2005). A type-2 fuzzy time-series model for stock index forecasting, Physica A, 353, pp. 445-462

Kosko, B. (1992). Neural Networks and Fuzzy Systems: A Dynamical Systems Approach to Machine Intelligence, Prentice Hall, pp. 323-333

Radeerom, M.; Srisa-an, C. and Kulthon Kasemsan, M. L. (2008). Prediction Method for Real Thai Stock Index Based on Neurofuzzy Approach, Trends in Intelligent Systems and Computer Engineering - Lecture Notes in Electrical Engineering, 6, pp. 327-347

Trifan, A.L. (2010). Financial time series forecasting using neural networks: A case study of the Bucharest Stock Exchange, Annals of DAAAM for 2010 \& Proceedings of the 21st International DAAAM Symposium, pp. 1381- 1383, ISBN 978-3-901509-73-5, ISSN 1726-9679

Yang, M.-S.; Hwang, P.Y.; Chen, D.H. (2004). Fuzzy clustering algorithms for mixed feature variables, Fuzzy Sets and Systems, 141, pp. 301-317 